\title{
A specifically designed aquatic exercise protocol to reduce chronic lower limb edema
}

\author{
Sergio Gianesini', Mirko Tessari', Primo Bacciglieri', \\ Anna Maria Malagoni', Erica Menegatti', Savino Occhionorelli', \\ Nino Basaglia ${ }^{3}$ and Paolo Zamboni'
}

\begin{abstract}
Objective: Despite the fact that muscle pump activation is known to positively impact chronic lower limb edema, objective measurements of standardized exercises for venous-lymphatic rehab are lacking. The aim of this investigation is to determine the effectiveness of an addressed physical activity exploiting the advantages of an aquatic environment.

Material and methods: Thirty-two lower limbs of 16 patients affected by bilateral chronic leg swelling were included ( 12 females, 4 males). All the patients underwent a protocol of five sessions of physical exercises specifically conceived inside a pool. Volumetry, subcutaneous thickness, ankle range of motion and symptomatology were assessed as outcome measures.

Results: One week after the end of the protocol, the average reduction in lower limb volume was $303.13 \pm 69.72 \mathrm{ml}$ $(p=0.00002)$ and $334.38 \pm 62.50 \mathrm{ml}(p=0.000003)$ in the right and left legs, respectively. Ankle range of motion and feeling of heaviness significantly improved.

Conclusion: A specifically designed aquatic protocol is able to positively impact chronic leg swelling offering a first line rehab for this medical condition.
\end{abstract}

\section{Keywords}

Chronic venous disease, chronic venous insufficiency, lymphoedema, venous stasis, calf muscle pump function

\section{Introduction}

Chronic edema of the lower limbs is a multifactorial condition that is mainly associated with failure of the venous and/or lymphatic systems. ${ }^{1} \mathrm{Up}$ to $30 \%$ of advanced chronic venous disease (CVD) conditions present with a concomitant lymphatic impairment, ${ }^{2}$ thus representing the most common cause of lower limb lymphedema in patients over 50 in westernized countries. ${ }^{3}$

Recent evidence has demonstrated that the incidence of venous lymphedema is often underestimated, considering the potential misdiagnosis among primary lymphedema and cases of iliac venous obstruction leading to secondary swelling. ${ }^{4}$ Properly collected epidemiologic data regarding chronic lower limb edema are lacking because of a general underestimation and misdiagnosis of the problem. ${ }^{5}$

Nevertheless, it is well known by health professionals how frequently this condition is encountered and how high the related economic costs are, even just considering, for example, that a prevalence of almost $50 \%$ has been reported in oncologic patients following radiotherapy. ${ }^{5}$ At the same time, the social, economic and health burden of CVD has been clearly stated in the literature, with up to $40 \%$ of Western populations with venous reflux and $2 \%$ of the National Health System expenses being dedicated to the pathology. ${ }^{6,7}$ Manual lymphatic drainage, meticulous skin care, pharmacological support, and compression devices represent the mainstay treatment for lymphedema. ${ }^{8}$

\footnotetext{
'Vascular Diseases Center, Unit of Translational Surgery, University of Ferrara, Italy

${ }^{2}$ Idrokinetik Clinic, Ferrara, Italy

${ }^{3}$ Department of Neuroscience and Rehabilitation, S. Giorgio Hospital, University of Ferrara, Italy
}

\section{Corresponding author:}

Sergio Gianesini, Vascular Diseases Center, Unit of Translational Surgery, University of Ferrara, Via Aldo Moro 8, Cona, Ferrara 44I00, Italy.

Email: sergiogianesini@hotmail.com 
Great importance has also been placed on the physical exercises used to mobilize interstitial fluid overload. Nevertheless, up until now, this last prophylactic and therapeutic approach has been deeply investigated only in upper limbs and in cancer patients who underwent related surgical and/or radiotherapy treatments. ${ }^{9}$ The plantar and calf muscle pumps play a fundamental role in the management of lower limb edema by stimulating both fluid reabsorption and proper venous hemodynamics. ${ }^{10}$

Comparisons among land-based and aquatic exercises have already demonstrated the great advantages of water activities brought by the exploitation of physical properties in other specialties' therapeutic and rehab scenarios. ${ }^{11-13}$ On the contrary, and to our knowledge, investigations regarding an objective assessment of the impact of water physical activity on chronic lower limb edema have yet to be done.

The primary endpoint of the present study is to evaluate the effect of a specifically designed aquatic exercise protocol on leg volume of hypomobile patients affected by chronic lower limb edema of venous and lymphatic origin. Secondary endpoints are the modification of the ankle range of motion, symptomatology and walking ability.

\section{Material and methods}

Thirty-two lower limbs of 16 patients affected by bilateral lymphedema were included in this prospective cohort study (12 females and 4 males; ages: $60 \pm 15$ years).

Fourteen cases were presented visible varices. According to the CEAP classification, ${ }^{14} 9$ legs were $\mathrm{C} 2,16$ were $\mathrm{C} 3,2$ were $\mathrm{C} 4$ and 1 was $\mathrm{C} 5$.

Two patients were affected by bilateral lymphedema secondary to surgical and radiotherapeutic oncologic protocols, without any venous drainage impairment.

Inclusion criteria were:

- Age $>18$ years old

- CVD

- Pitting edema and/or positive Kaposi-Stemmer sign (inability to pinch a fold of skin on the dorsum of the base of the second toe). ${ }^{15}$

- Lower limb edema onset $>3$ months

\section{Exclusion criteria:}

- previous varicose vein procedures

- previous lower limb trauma

- heart/respiratory/renal failure

- rheumatologic disease and

- fear of water.
All the participants gave their full informed consent before entering in the study; institutional review board approval was obtained. All the patients underwent a protocol of physical exercises specifically conceived inside a pool and designed to maximize the ankle motion together with the calf and plantar muscle pumps. The pool had a depth of $120 \mathrm{~cm}$ and an area of $16 \mathrm{~m}^{2}$. It was endowed with an anti-slip pavement and a handrail. The water was not thermal and at a constant temperature of $33^{\circ} \mathrm{C}$. $\mathrm{pH}$, chloride concentration and water clarity were checked daily.

The physical exercise protocol was named "HydroFE" and it included the following activities:

- warming up by a cycling-like activity in a supine position while holding the handrail

- tip-toe exercise (four series of 10 repetitions each)

- hip flexion-extension on the non-weight bearing limb (four series of 10 repetitions each)

- tip-toe exercise on a step (four series of 10 repetitions each)

- knee flexion-extension at increasing velocity (four series of 10 repetitions each)

- forward and backward walking for $5 \mathrm{~min}$

- lateral walking on both sides for $5 \mathrm{~min}$

- cycling-like single push while standing up on the contralateral limb, holding the kickboard for balance. The exercise is done without moving the ankle joint (four series of 10 repetitions each)

- ankle flexion-extension while keeping the knee bent $90^{\circ}$ (four series of 10 repetitions each)

- cool-down by a cycling-like activity in a supine position while holding the handrail.

The protocol was performed under the supervision of a hydrotherapeutic personal trainer who was present in the water with two patients at a time. The patients repeated the exercises two sessions a week $(50 \mathrm{~min}$ each), for a total of five sessions.

Right before starting the first session and one week after the end of the last session, the entire study group underwent a high-resolution echo-color-Doppler scanning (Esaote My Lab 25; 8-12 MHz linear transducer) to evaluate both the venous drainage and the subcutaneous tissue thickness (distance from the muscular fascia to the skin layer). ${ }^{16}$ All the patients were assessed in a standing position, testing the deep, superficial and perforator systems, evoking the flow by manual compression/relaxation.

The points of scanning and measurements were as follows:

B: middle lower third of the leg at the ankle level

B1: posterior middle third of the leg

C: medial middle third of the leg 
D: medial upper third of the leg

E: below the knee

F: medial middle third of the thigh

G: medial upper third of the thigh.

At the same specific points, circumferences were assessed by a centimetre-tape right before and one week after the end of the five sessions. At the same time, the active range of motion of the ankle (ROM) was determined in all legs having the patients sit with the foot parallel to the ground and measuring the grade of dorsiflexion and plantar flexion by a goniometer. ${ }^{16-18}$

The limb volumetry was assessed by water plethysmography before and at the end of every single session. ${ }^{19}$ One week after the end of the protocol, a $100 \mathrm{~mm}$ visual analogue scale (VAS) was used for assessing the patients' feeling of lower limb heaviness. ${ }^{20}$ A functional ambulation classification (FAC) assessment was performed at the beginning and at the end of the five sessions in order to determine the walking abilities of all the patients. ${ }^{21}$ All the measures were taken in the same room, at the same temperature, collecting the data in the same seasonal period, in between 8 and 9 a.m. The sonographic data were collected by the same physician, highly skilled in ultrasonography. The volumetry, circumference, ROM, VAS, and FAC data were obtained by a physician with as $\mathrm{PhD}$ in vascular medicine.

\section{Statistical analysis}

InStat GraphPad (GraphPad Software, Inc.La Jolla, CA 92037 USA) was used for statistical analysis. The data were expressed as mean \pm standard deviation. In order to verify the data distributions, KolmogorovSmirnov test was used. The differences in leg volume pre- and post-round were evaluated by Student's $t$-test and Wilcoxon test as appropriate. Pearson's correlation coefficient was calculated to identify the correlation between leg volume, circumference, subcutaneous thickness, and ROM. Statistical significance was defined as $p<0.05$.

\section{Results}

All the patients completed the protocol without major and minor complications. One week after the end of the protocol, water plethysmography reported a significant average reduction in all lower limbs' volume, decreasing from $2950 \pm 722 \mathrm{ml}$ to $2688 \pm 624 \mathrm{ml}(p=0.00002)$ and from $2837 \pm 689 \mathrm{ml}$ to $2541 \pm 526 \mathrm{ml}(p=0.000003)$ in the right and left legs, respectively (Figure 1).

At the end of every single session, in comparison with the previous session, a significant volume

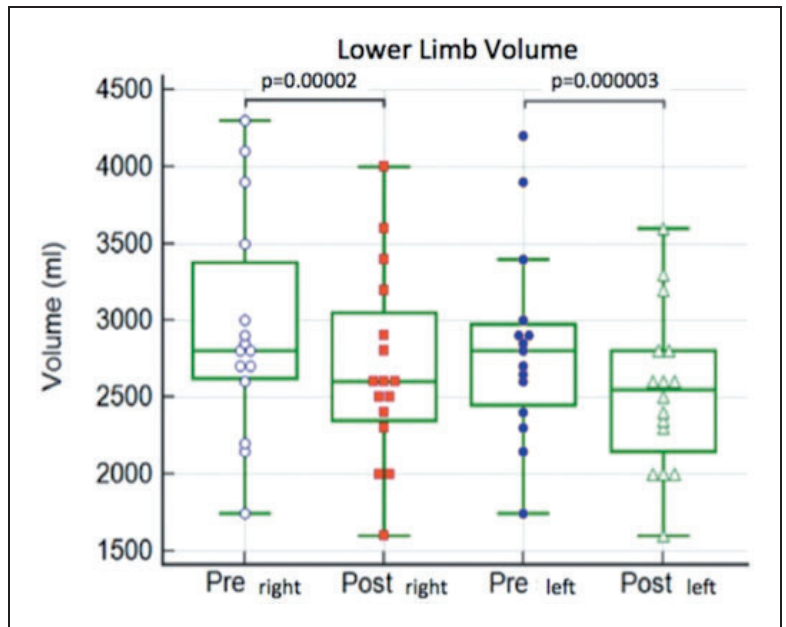

Figure I. Volumes of lower limb before and after the rehab session.

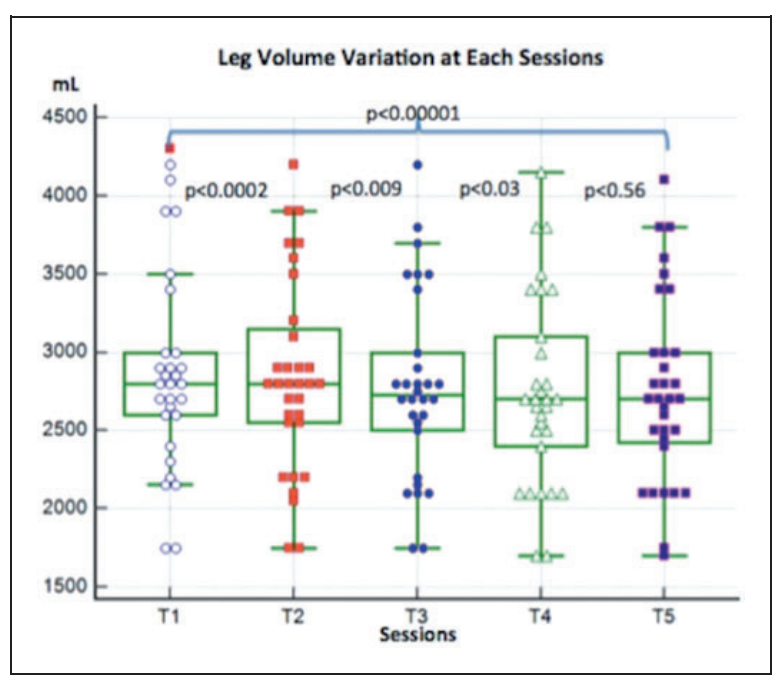

Figure 2. Volume variation of lower limb at each rehab session.

reduction was reported (Figure 2), with the exception of the volume variation among the fourth and fifth sessions. A significant volume reduction was reported among the baseline and the final assessment for each single patient (Figure 3).

One week after the end of the protocol, the subcutaneous skin thickness and the circumferences significantly decreased in the assessment points $(p<0.0001)$ (detailed data are reported in Table 1). Venous reflux was detected in the superficial venous system of all the 14 cases affected by visible varices. Comparing the assessments done before and after the five sessions of aquatic exercise, no significant differences were reported in the reflux time. At the same observation time, the ROM measurement reported a significant increase both in the dorsal and plantar flexion of the 


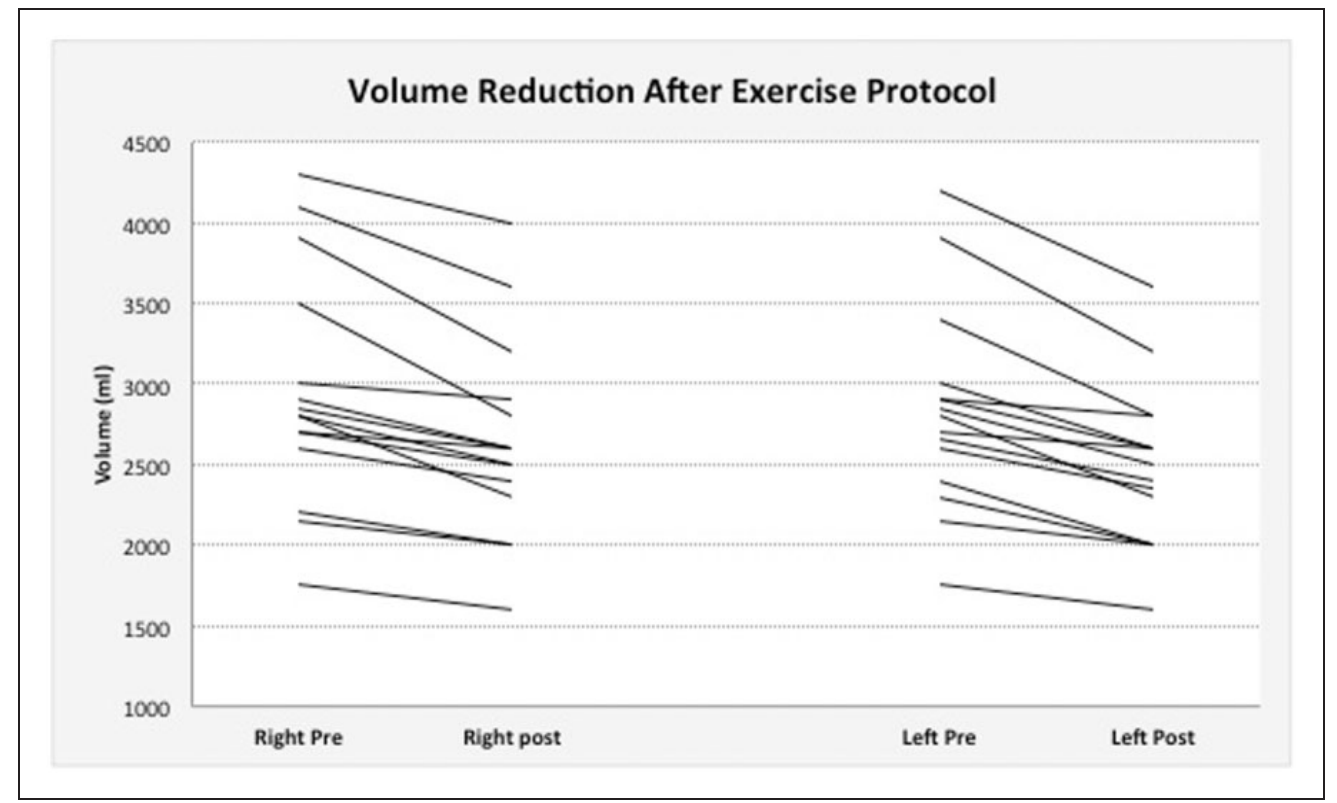

Figure 3. Leg volume reduction after five sessions of exercise. The black lines represent each patient's right and left legs.

Table I. Lower limb circumference, subcutaneous thickness and ankle ROM.

\begin{tabular}{|c|c|c|c|c|c|c|}
\hline & \multicolumn{6}{|c|}{ Circumference $(\mathrm{cm})$} \\
\hline & $B$ & $B I$ & $C$ & $D$ & $F$ & $G$ \\
\hline \multirow[t]{2}{*}{ Right leg } & -2.63 & -2.88 & -2.41 & -1.25 & -1.38 & -2.19 \\
\hline & $(p=0.000 \mathrm{I})$ & $(p<0.0001)$ & $(p<0.0001)$ & $(p=0.000 \mathrm{I})$ & $(p<0.0001)$ & $(p<0.000 \mathrm{I})$ \\
\hline \multirow[t]{4}{*}{ Left leg } & -1.27 & -2.03 & -1.34 & -1.03 & -1.34 & -2.00 \\
\hline & $(p=0.000 \mathrm{I})$ & $(p<0.0001)$ & $(p<0.000 \mathrm{I})$ & $(p<0.000 \mathrm{I})$ & $(p<0.000 \mathrm{I})$ & $(p=0.000 I)$ \\
\hline & \multicolumn{6}{|c|}{ Subcutaneous thickness $(\mathrm{cm})$} \\
\hline & Foot dorsum & $B$ & $C$ & $D$ & $F$ & $G$ \\
\hline \multirow[t]{2}{*}{ Right leg } & -3.37 & -6.40 & -381 & -4.03 & -3.39 & -3.64 \\
\hline & $(p=0.001)$ & $(p=0.0006)$ & $(p=0.002)$ & $(p=0.004)$ & $(p=0.004)$ & $(p=0.02)$ \\
\hline \multirow[t]{4}{*}{ Left leg } & -3.39 & -5.42 & -4.28 & -3.69 & -3.15 & -2.84 \\
\hline & $(p=0.003)$ & $(p=0.000 \mathrm{I})$ & $(p=0.0002)$ & $(p=0.0007)$ & $(p=0.0004)$ & $(p=0.008)$ \\
\hline & \multicolumn{6}{|c|}{ Ankle ROM increase $\left({ }^{\circ}\right)$} \\
\hline & \multicolumn{3}{|l|}{ Dorsiflexion } & \multicolumn{3}{|l|}{ Plantar flexion } \\
\hline Right & \multicolumn{3}{|c|}{$2.5^{\circ}, p=0.003$} & \multicolumn{3}{|c|}{$2.7^{\circ}, p=0.001$} \\
\hline Left & \multicolumn{3}{|c|}{$4.1^{\circ}, p=0.0001$} & \multicolumn{3}{|c|}{$2.18^{\circ}, p=0.001$} \\
\hline
\end{tabular}

ROM: range of motion of the ankle.

ankle, both on the right (dorsal $2.5^{\circ}, p=0.003$; plantar $2.8^{\circ}, p=0.001$ ) and left sides (dorsal $4.1^{\circ}, p=0.0001$; plantar $\left.2.2^{\circ}, p=0.001\right)$. A direct correlation was found among the assessed volume and the circumference values in B point (right leg: $r^{2}=0.88, p<0.0001$; left leg: $\left.r^{2}=0.90, p<0.0001\right)$, the subcutaneous thickness (right leg: $r^{2}=0.85, p<0.0001$; left leg: $r^{2}=0.71$, $p<0.0001$ ) and the ankle ROM (right leg: $r^{2}=0.81$, $p<0.002$; left leg: $r^{2}=0.71, p<0.0001$ ).

VAS evaluation reported a significant change in the lower limb heaviness feeling of the patient, decreasing from $7.3 \pm 2.1$ to $5.5 \pm 1.6(p=0.001)$. There was no variation in the FAC assessment at the completion of the protocol. 


\section{Discussion}

Chronic lower leg swelling represents an underestimated and undertreated pathological condition afflicting almost one-third of CVD patients and up to $80 \%$ of obese and hypomobile people. The costs related to this medical condition are as alarming as the potential evolution of the pathology toward infection, disfigurement, and disability. Based on this information alone, early therapeutic intervention becomes mandatory. ${ }^{22,23}$ Venous and lymphatic stasis management includes physical exercises aimed to maximize the interstitial fluid reabsorption by calf and plantar pump activation. ${ }^{24}$ Nevertheless, this option can become quite cumbersome in cases of advanced age, hypomobility, arthrosis and obesity: all conditions that are frequently associated with chronic leg swelling. Aquatic exercises have already demonstrated benefits induced by the physics properties of water.

Water density, temperature, hydrostatic pressure and buoyancy are all features that can be exploited to work on effects related to gravity, resistance and joint impact. Moreover, this medium stimulates both the neuromuscular and metabolic systems, positively impacting also the psychological side. ${ }^{25,26}$ Moreover, the same water temperature has its role. The recommended temperature ranges from $29^{\circ} \mathrm{C}$ (vigorous activities) and $33^{\circ} \mathrm{C}$ (light activities like walking). Exercising moderately below $31^{\circ} \mathrm{C}$ should be avoided in order not to trigger shivering and muscle spasms. ${ }^{27}$

For these intrinsic and extrinsic features, water therapy is already widely used in orthopedic, ${ }^{28}$ rheumatologic, ${ }^{29}$ neurologic, ${ }^{30}$ psychologic ${ }^{31}$ and pneumologic rehabilitation protocols. ${ }^{32}$ According to Stevin's law, the pressure at a point in a liquid in static equilibrium depends only on the depth at that point $\left(\mathrm{P}=\mathrm{P}_{0}+\mathrm{dgh}\right.$; $\mathrm{P}_{0}=$ external pressure on the surface, $\mathrm{h}=$ depth, $\mathrm{g}=$ gravity acceleration, $\mathrm{d}=$ liquid density). ${ }^{33}$ Consequently, the hydrostatic pressure of water gradually increases with greater depth $(0.74 \mathrm{mmHg}$ every $\mathrm{cm})$, so favoring limb drainage.

Trans Mural Pressure (TMP) represents a fundamental parameter in both venous and lymphatics systems impairment. TMP is expressed as a difference among the pressure inside the vessel and the one outside the conduit. The internal and external pressure exert their opposite actions on the inner/outer vessel walls, determining its degree of dilation, according to the Laplace Law $\left(\right.$ wall tension/vessel radius $=$ pressure gradient) ${ }^{33}$

Considering that into the water a gradual pressure increase is developed from the surface to the depth of the pool (Stevin's Law), aquatic activities exploit this graduated external pressure on the leg to favor both venous and lymphatic drainage. ${ }^{34}$ Viscosity provides a resistance that promotes strengthening of the calf muscular action on the venous and lymphatic systems.
These properties have led to the use of water activities for lower limb drainage since ancient times. ${ }^{35}$ Nevertheless, literature is reporting data mainly regarding the effect of general balneotherapy activities on advanced CVD cases, showing an improvement in trophic skin lesions and in patients' quality of life. ${ }^{36}$

Despite an increasing diffusion of commercial SPA programs claiming effectiveness on lower limb drainage, objective data are lacking regarding the outcome of aquatic exercises on venous and lymphatic return. Plantar and/or calf muscle pumps strictly influence venous blood and lymph return, and their structured land-based exercise has already demonstrated to positively impact the drainage. ${ }^{37}$ Nevertheless, these kinds of activity results are not always feasible in the majority of patients because of hypomobility, obesity and joint issues.

Water environments facilitate and maximize both muscular activation and joint mobilization by removing part of the gravitational load while exerting a favorable external compression. Ankle joint functionality constitutes a fundamental mainstay in the venous blood and lymph propulsion, and its ROM decrease has been already identified as a risk factor for venous drainage impairment. ${ }^{38}$ The specifics of this protocol focus on joint mobilization with a particular focus on the ankle. In particular, backwards walking has recently been demonstrated to be associated with a main propulsion action of the ankle, so maximizing its role in drainage promotion. ${ }^{39}$ At the same time, lateral walking was used to maximize ankle joint movement in all the different planes. ${ }^{40}$ The objective and significantly positive outcome of this protocol is found in the reduction of lower limb volume. Our data demonstrate how a structured aquatic exercise protocol is associated with a reduction in chronic lower limb edema and with an increase in the directly related ankle mobility.

The direct correlation among this parameter with both leg circumferences and subcutaneous tissue, together with the improvement reported in the patient symptomatology, represents the evidence of the effectiveness of this exercise protocol in reducing the severity of chronic lower limb edema. The sub-analysis of the volume variation at every session demonstrates the maintenance of the effect over time, with a progressive drainage improvement. The importance of compliance to the maintenance phase of the chronic swelling treatment is fundamental. ${ }^{4,8}$ Water activities have demonstrated a good compliance and a positive impact also on the psychological aspect, so positively influencing the same compliance rate. Moreover, the objective outcomes associated with the proposed protocol offer a cost-effective first-line therapeutic approach for primary care physicians who are daily challenged to find an effective management for chronic lower leg edema. 
Further studies should be addressed to compare the same exercise protocol on land, specifically assessing the eventual effects on the lymphatics and venous circulation per se. Other investigations are also needed to determine the optimum temperature for veno-lypmhatic drainage facilitation, to include a detailed analysis of the impact on patient quality of life and to establish the exact timeline for an enduring drainage improvement by water activities. These kinds of assessments could finally give the needed scientific value to all those aquatic protocols that, up to now, in the SPA world are claiming venous and lymphatics effects without corresponding objective data in the literature.

\section{Acknowledgments}

We are deeply grateful to Mattia Guerzoni and Idrokinetik Clinic who provided the hydrotherapic pool at no cost.

\section{Authors' contribution}

SG conceived and designed the study, researched literature and collected data and wrote the first draft of the manuscript; MT was involved in data collection and data analysis; PB was involved in protocol development and critical review of the manuscript; AMM and EM were involved in manuscript preparation, data analysis and statistical analysis; SO was involved in data collection and critical review of the manuscript; NB was involved in protocol development and critical review of the manuscript and $\mathrm{PZ}$ was involved in protocol development, patient recruitment and data analysis, manuscript critical review and preparation. All authors reviewed and edited the manuscript and approved the final version of the manuscript.

\section{Declaration of Conflicting Interests}

The author(s) declared no potential conflicts of interest with respect to the research, authorship, and/or publication of this article.

\section{Funding}

The author(s) received no financial support for the research, authorship, and/or publication of this article.

\section{References}

1. Stout N, Partsch H, Szolnoky G, et al. Chronic edema of the lower extremities: international consensus recommendations for compression therapy clinical research trials. Int Angiol 2012; 31: 316-329.

2. Bunke N, Brown K and Bergan J. Phlebolymphedema: usually unrecog- nized, often poorly treated. Perspect Vasc Surg Endovasc Ther 2009; 21: 65-68.

3. Raju S, Furrh JB 4th and Neglén P. Diagnosis and treatment of venous lymphedema. J Vasc Surg 2012; 55: $141-149$.

4. Moffatt CJ, Franks PJ, Doherty DC, et al. Lymphoedema: an underestimated health problem. $Q J$ Med 2003; 96: $731-738$.
5. Williams AF1, Franks PJ and Moffatt CJ. Lymphoedema: estimating the size of the problem. Palliat Med 2005; 19: 300-313.

6. Milic DJ. Prevalence and socioeconomic data in chronic venous disease: how useful are they in planning appropriate management? Medicographia 2011; 33: 253-258.

7. McLafferty RB, Passman MA, Caprini JA, et al. Increasing awareness about venous disease: The American Venous Forum expands the National Venous Screening Program. J Vasc Surg 2008; 48: 394-399.

8. International Society of Lymphology. The diagnosis and treatment of peripheral lymphedema: 2013 Consensus Document of the International Society of Lymphology. Lymphology 2013; 46: 1-11.

9. Johansson K, Hayes S, Speck RM, et al. Water-based exercise for patients with chronic arm lymphedema: a randomized controlled pilot trial. Am J Phys Med Rehabil 2013; 92: 312-319.

10. Goddard AA1, Pierce CS and McLeod KJ. Reversal of lower limb edema by calf muscle pump stimulation. J Cardiopulm Rehabil Prev 2008; 28: 174-179.

11. Rahmann EA. Exercise for people with hip or knee osteoarthritis: a comparison of land-based and aquatic interventions. Open Access J Sports Med 2010; 1: 123-135.

12. Wang T, Belza B, Elaine Thompson F, et al. Effects of aquatic exercise on flexibility, strength and aerobic fitness in adults with osteoarthritis of the hip or knee. $J A d v$ Nurs 2007; 57: 141-152.

13. Lee JH and Sung E. The effects of aquatic walking and jogging program on physical function and fall efficacy in patients with degenerative lumbar spinal stenosis. $J$ Exerc Rehabil 2015; 30: 272-275.

14. Rabe E and Pannier F. Clinical, aetiological, anatomical and pathological classification (CEAP): gold standard and limits. Phlebology 2012; 27: 114-118.

15. Stemmer R. Ein klinisches zeichen zur gruhund differential diagnose des lymphodems. [A clinical symptom for the early and differential diagnosis of lymphedema]. German. Vasa 1976; 5: 261-262.

16. Suehiro K, Morikage N, Murakami M, et al. Subcutaneous tissue ultrasonography in legs with dependent edema and secondary lymphedema. Ann Vasc Dis 2014; 7: 21-27.

17. Brosseau L, Tousignant M, Budd J, et al. Intratester and intertester reliability and criterion validity of the parallelogram and universal goniometers for active knee flexion in healthy subjects. Physiother Res Int 1997; 2: 150-166.

18. Konor MM, Morton S, Eckerson JM, et al. Reliability of three measures of ankle dorsiflexion range of motion. Int J Sports Phys Ther 2012; 7: 279-287.

19. Rabe E, Stücker M and Ottillinger B. Water displacement leg volumetry in clinical studies-a discussion of error sources. BMC Med Res Methodol 2010; 13: 10:5.

20. Quinn JV and Wells GA. An assessment of clinical wound evaluation scale. Acad Emerg Med 1998; 5: 583-586.

21. Viosca E, Martínez JL, Almagro PL, et al. Proposal and validation of a new functional ambulation classification 
scale for clinical use. Arch Phys Med Rehabil 2005; 86: 1234-1238.

22. Keast DH, Despatis M, Allen JO, et al. Chronic oedema/ lymphoedema: under-recognised and under-treated. Int Wound $J$ 2015; 12: 328-333.

23. Ridner SH. The psycho-social impact of lymphedema. Lymphat Res Biol 2009; 7: 109-112.

24. Ely JW, Osheroff JA, Chambliss ML, et al. Approach to leg edema of unclear etiology. J Am Board Fam Med 2006; 19: 148-160.

25. Torres-Ronda L and Del Alcázar XS. The properties of water and their applications for training. J Hum Kinet 2014; 44: 237-248.

26. Mooventhan A and Nivethitha L. Scientific evidencebased effects of hydrotherapy on various systems of the body. N Am J Med Sci 2014; 6: 199-209.

27. Nakanishi Y, Kimura T and Yokoo Y. Maximal physiological responses to deep water running at thermoneutral temperature. Appl Human Sci 1999; 18: 31-35.

28. Barker AL, Talevski J, Morello RT, et al. Effectiveness of aquatic exercise for musculoskeletal conditions: a metaanalysis. Arch Phys Med Rehabil 2014; 95: 1776-1786.

29. Verhagen AP, Bierma-Zeinstra SM, Boers M, et al. Balneotherapy (or spa therapy) for rheumatoid arthritis. Cochrane Database Syst Rev 2015; 4: CD000518.

30. Frohman AN, Okuda DT, Beh S, et al. Aquatic training in MS: neurotherapeutic impact upon quality of life. Ann Clin Transl Neurol 2015; 2: 864-872.

31. Latorre-Román PÁ, Rentero-Blanco M, LaredoAguilera JA, et al. Effect of a 12-day balneotherapy programme on pain, mood, sleep, and depression in healthy elderly people. Psychogeriatrics 2015; 15: 14-19.
32. RJ1 McNamara, McKeough ZJ, McKenzie DK, et al. Water-based exercise training for chronic obstructive pulmonary disease. Cochrane Database Syst Rev 2013; 12: CD008290.

33. Franceschi C, Cappelli M, Ermini S, et al. CHIVA: hemodynamic concept, strategy and results. Int Angiol 2015; 35: 8-30.

34. H1 Noddeland and Winkel J. Effects of leg activity and ambient barometric pressure on foot swelling and lowerlimb skin temperature during $8 \mathrm{~h}$ of sitting. Eur $J$ Appl Physiol Occup Physiol 1988; 57: 409-414.

35. Gehrke A, Hussain M, Kleinschmidt $J$, et al. Hydrotherapy in swellings with special reference to lymphedema. Z Lymphol 1981; 5: 100-106.

36. Carpentier PH, Blaise S, Satger B, et al. A multicenter randomized controlled trial evaluating balneotherapy in patients with advanced chronic venous insufficiency. J Vasc Surg 2014; 59: 447-454.e1.

37. Padberg FT Jr, Johnston MV and Sisto SA. Structured exercise improves calf muscle pump function in chronic venous insufficiency: a randomized trial. J Vasc Surg 2004; 39: 79-87.

38. Shiman MI, Pieper B, Templin TN, et al. Venous ulcers: A reappraisal analyzing the effects of neuropathy, muscle involvement, and range of motion upon gait and calf muscle function. Wound Repair Regen 2009; 17: 147-152.

39. Hoogkamer W1, Meyns P and Duysens J. Steps forward in understanding backward gait: from basic circuits to rehabilitation. Exerc Sport Sci Rev 2014; 42: 23-29.

40. Donelan JM1, Shipman DW, Kram R, et al. Mechanical and metabolic requirements for active lateral stabilization in human walking. $J$ Biomech 2004; 37: 827-835. 